\title{
Protein tyrosine phosphatase receptor-type $O$ expression as a prognostic marker in patients with acute coronary syndrome undergoing percutaneous coronary intervention: A prospective study
}

\author{
MINGHUI HAO, MING GUO and RUI YAN \\ Department of Cardiology, Beijing Luhe Hospital, Capital Medical University of Medical Sciences, \\ Beijing 101100, P.R. China
}

Received July 21, 2020; Accepted December 10, 2020

DOI: $10.3892 /$ etm.2021.9852

\begin{abstract}
Optimal clinical indicators are crucial for evaluating the prognosis of patients with acute coronary syndrome (ACS). In the present study, the potential prognostic value of protein tyrosine phosphatase receptor-type O (PTPRO) expression in the peripheral blood mononuclear cells of patients with ACS undergoing percutaneous coronary intervention (PCI) was investigated. Patients diagnosed with ACS were prospectively recruited, and PTPRO expression in mononuclear cells separated from peripheral blood was assessed by western blotting. The prognosis was judged by the occurrence of major adverse cardiovascular events. Cox regression analyses were performed to assess the association between PTPRO expression and prognosis. In the enrolled 185 patients with ACS, PTPRO expression was lower after PCI compared with that before PCI $(\mathrm{P}<0.05)$. Although the pre-PCI PTPRO expression did not differ significantly between the good and poor prognosis
\end{abstract}

Correspondence to: Dr Ming Guo, Department of Cardiology, Beijing Luhe Hospital, Capital Medical University of Medical Sciences, 82 Xinnan Road, Tongzhou, Beijing 101100, P.R. China E-mail: guoming0318@sina.com

Abbreviations: ACS, acute coronary syndrome; PCI, percutaneous coronary intervention; MACE, major adverse cardiovascular event; PTPRO, protein tyrosine phosphatase receptor-type O; TLR4, Toll-like receptor 4; NF- $\mathrm{B}$, nuclear factor $\kappa \mathrm{B}$; BMI, body mass index; STEMI, ST-elevation acute myocardial infarction; NSTEMI, non-ST-elevation acute myocardial infarction; UA, unstable angina; RMI, recurrent myocardial infarction; RUA, recurrent unstable angina; TVR, target vessel revascularization; RIPA, radioimmunoprecipitation assay; SDS, sodium dodecyl sulfate; PAGE, polyacrylamide gel electrophoresis; PBST, phosphate-buffered saline containing Tween 20; ECL, enhanced chemiluminescence; ROC, receiver operating characteristic; AUC, area under the ROC curve; CI, confidence interval

Key words: ACS, PCI, peripheral blood mononuclear cells, PTPRO, prognosis groups, PTPRO expression after PCI was significantly lower in the good prognosis group compared with the poor prognosis group $(\mathrm{P}<0.05)$. The area under the receiver operating characteristic curve for the prognostic value of post-PCI PTPRO expression was significantly greater than that for pre-PCI PTPRO expression $(\mathrm{P}<0.05)$. Cox regression analysis identified high post-PCI PTPRO expression as an independent risk factor for poor prognosis in patients with ACS $(\mathrm{P}<0.05)$, and further analysis indicated that the post-PCI PTPRO expression level was associated with the prognosis of patients with ACS $(\mathrm{P}<0.05)$. PTPRO expression in peripheral blood mononuclear cells after PCI is associated with the prognosis of patients with ACS, with high PTPRO expression indicating a high risk of poor prognosis in patients with ACS.

\section{Introduction}

With the aging of the population and the quickening pace of life, the incidence of acute coronary syndrome (ACS) has continued to increase (1). ACS is a common cardiovascular disease with a high morbidity and mortality rates (2). Although the risks of myocardial ischemia and hypoxia have been improved in patients with ACS undergoing percutaneous coronary intervention (PCI), some patients still experience major adverse cardiovascular events (MACEs) (3). There is an urgent need for indicators that can predict the occurrence of MACEs in patients with ACS undergoing PCI with high sensitivity and specificity.

Protein tyrosine phosphatase receptor-type O (PTPRO), a protein tyrosine phosphatase receptor, has been reported to be involved in the pathogenesis and progression of lung squamous cell carcinoma (4), breast cancer (5), colorectal cancer (6) and hepatocellular carcinoma (7). However, few studies have focused on the association between PTPRO and ACS. The Toll-like receptor 4 (TLR4)/nuclear factor $\mathrm{B}(\mathrm{NF}-\kappa \mathrm{B})$ signaling pathway, which plays an important role in the inflammatory response, has been reported to be involved in the development of ACS $(8,9)$. Through the TLR4/NF- $\kappa$ B signaling pathway, the expression of PTPRO was shown to aggravate the inflammatory response in ulcerative colitis (10) and to regulate oxidative stress and apoptosis (11). Based on this association 
between PTPRO expression and the inflammatory response, it was hypothesized that PTPRO expression might be associated with the progression of ACS. Therefore, the present study was conducted to explore the association between the expression level of PTPRO in peripheral blood mononuclear cells and the prognosis of patients with ACS undergoing PCI.

\section{Materials and methods}

Patients. A total of 185 patients (age range, 31-78 years; 130 males and 55 females) with ACS admitted to Beijing Luhe Hospital, Capital Medical University (Beijing, China) between April 2016 and April 2017 were enrolled in this prospective study. ACS was diagnosed according to the Guidelines for the Rapid Diagnosis and Treatment of ACS in the Emergency Department (12). The inclusion criteria were as follows: i) Age $\leq 80$ years; ii) first diagnosis with ACS; iii) planned treatment with PCI; iv) hospital admission within $24 \mathrm{~h}$ after ACS onset; and v) regular use of anti-platelet aggregation drugs after PCI. Patients were excluded if they: i) Had contraindications for PCI; ii) underwent coronary artery bypass grafting; iii) had lower extremity arteriosclerosis obliterans, stroke, chronic obstructive pulmonary disease, hyperhomocysteinemia, heart valvular disease or dilated cardiomyopathy, cancer, autoimmune diseases or inflammatory diseases, liver fibrosis, or severe liver or renal dysfunction; or iv) were lost during the follow-up period. The study was approved by the medical ethics committee of Beijing Lube Hospital, Capital Medical University, and all patients included this study provided written informed consent.

Data collection. The following patient data were collected: Age, sex, body mass index (BMI), medical history (such as hypertension, diabetes, hyperlipidemia), family history of ACS, location and number of vascular lesions, and clinical type of ACS [including ST-elevation acute myocardial infarction (STEMI), non-ST-elevation acute myocardial infarction (NSTEMI), and unstable angina (UA)].

The 36 months of follow-up included examination at 1 month after discharge and once every 3 months thereafter. The prognosis of patients with ACS undergoing PCI was evaluated by telephone follow-up survey and readmission. A poor prognosis was considered when a MACE occurred, including recurrent myocardial infarction (RMI), recurrent unstable angina (RUA), stent restenosis, target vessel revascularization (TVR), and cardiac death. The endpoints were the occurrence of MACEs or the end of follow-up.

Measurement of PTPRO expression. A total of $10 \mathrm{ml}$ fasting venous blood was extracted from patients with ACS within $24 \mathrm{~h}$ after admission and again 7 days after PCI. Mononuclear cells were isolated from peripheral blood by Ficoll density gradient centrifugation (13).

The expression level of PTPRO in mononuclear cells was detected by western blotting (14). Briefly, radioimmunoprecipitation assay (RIPA) buffer was added to the test tube containing mononuclear cells to lyse the cells, and then the proteins were isolated and quantified using a BCA protein Quantitative kit (cat. no. KA3718; Abnova). The proteins were separated by $10 \%$ sodium dodecyl sulfate (SDS)-polyacrylamide gel electrophoresis (PAGE) at $120 \mathrm{~V}$ until bromophenol blue ran out of the gel surface, and the PVDF membrane transfer process was performed with $300 \mathrm{~mA}$ current for $60 \mathrm{~min}$ after balancing with the membrane buffer three times. Sealing was achieved by immersion in 5\% skim milk for $1 \mathrm{~h}$ at room temperature. The membrane was washed three times with phosphate-buffered saline containing Tween-20 (PBST) for 5 min each time, and then incubated with rabbit anti-PTPRO polyclonal (1:1,000; cat. no. H00005800-W01P; Abnova) or rabbit anti- $\beta$-actin (1:500; cat. no. PAB29054; Abnova) antibodies overnight at $4^{\circ} \mathrm{C}$. The membrane was again washed three times with PBST for 10 min each time, after which goat anti-rabbit IgG secondary antibodies (1:5,000; cat. no. A21020; Yakoyin Biotechnology Co., Ltd.) was added for incubation for $1 \mathrm{~h}$ at room temperature. The membrane was again washed three times with PBST for 10 min each time, and then exposure was achieved by enhanced chemiluminescence (ECL). Gray-scale values were calculated using ImageJ 1.8.0 software (National Institute of Health), and relative expression levels were calculated by normalization to $\beta$-actin expression.

Statistical analysis. R 3.6.3 software (https://www.r-project. org/) was applied for data analysis, and $\mathrm{P}<0.05$ was considered statistically significant. A paired sample t-test was used for the intra-group comparison of normal distribution, and an independent sample t-test was used for the comparison between two groups. The measurement data with a normal distribution are expressed as mean \pm standard deviation $(\bar{x} \pm s)$. Mixed ANOVA model was used for comparisons in PTPRO before and after PCI between groups. The count data were expressed as rate [n (\%)], and the $\chi^{2}$ test was used for comparison between groups. Receiver operating characteristic (ROC) curve analysis was applied to estimate the efficacy of PTPRO levels for predicting the prognosis of patients with ACS undergoing PCI. Kaplan-Meier analysis was employed to draw disease-free survival curves for the high PTPRO groups and low PTPRO groups, and the curves were compared using the log-rank method. Moreover, Cox regression analysis was performed to analyze risk factors for poor prognosis among patients with ACS undergoing PCI, and the Cox regression analysis with restrictive cubic spline model was used to evaluate the relationship between the expression level of PTPRO and the prognosis of patients with ACS undergoing PCI.

\section{Results}

Prognosis of patients. A total of 185 patients with ACS aged 31-78 years were enrolled in the present study, including 130 men and 55 women. During the 36 months of follow-up, 60 patients with ACS suffered from MACEs (32.43\%), including RMI in 26 patients (14.05\%), RUA in 14 patients (7.57\%), stent restenosis in 12 patients $(6.49 \%)$, TVR in 5 patients $(2.70 \%)$, and cardiac death in 3 patients $(1.62 \%)$.

Comparison of PTPRO expression levels between patients with poor and good prognoses. According to the occurrence of MACEs during the follow-up, patients with ACS were divided into two groups: Good prognosis group $(n=125)$ and poor prognosis group $(n=60)$. The PTPRO expression level after PCI in the good prognosis group was significantly lower compared 
Table I. Comparison of PTPRO expression levels in patients with ACS according to prognosis.

\begin{tabular}{lcccr}
\hline Group & $\mathrm{N}$ & PTPRO before PCI $(\bar{x} \pm s)$ & PTPRO after PCI $(\bar{x} \pm s)$ & $t$ \\
\hline Poor prognosis group & 60 & $1.04 \pm 0.13$ & $0.47 \pm 0.09$ & 29.133 \\
Good prognosis group & 125 & $1.02 \pm 0.13$ & $0.34 \pm 0.07$ & 56.543 \\
$T$ & & 0.841 & 9.918 & $<0.001$ \\
P-value & & 0.401 & $<0.001$ & \\
\hline
\end{tabular}

PTPRO, protein tyrosine phosphatase receptor-type O; PCI, percutaneous coronary intervention.

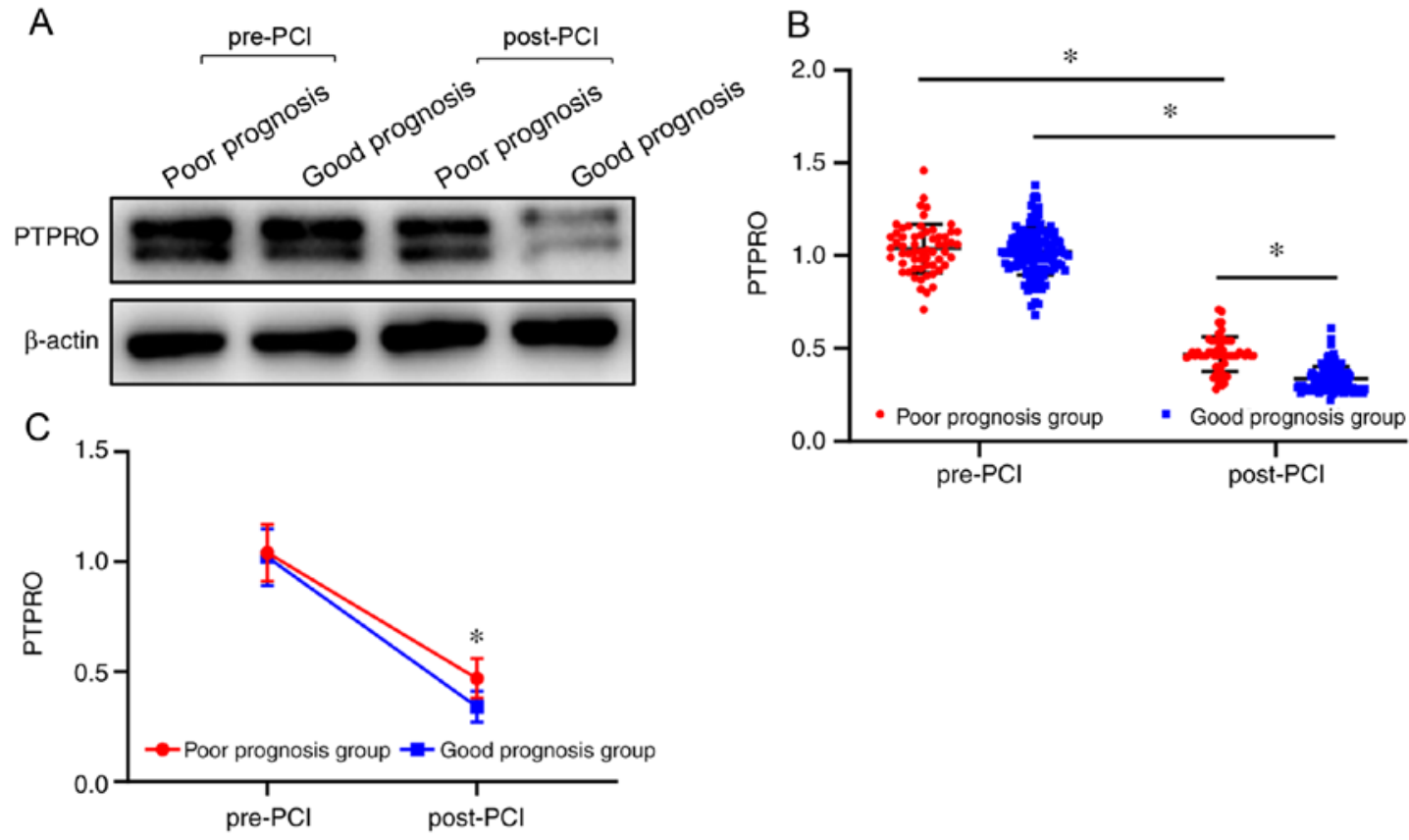

Figure 1. PTPRO expression levels in peripheral blood mononuclear cells of patients with acute coronary syndrome before and after PCI, and according to prognosis. PTPRO expression. (A) Western blotting images of different groups. (B) Quantitative analysis of protein in different groups. (C) comparison of PTPRO expressions between different groups. ${ }^{*} \mathrm{P}<0.05$. PTPRO, protein tyrosine phosphatase receptor-type O; PCI, percutaneous coronary intervention.

with that of the poor prognosis group ( $\mathrm{P}<0.05$; Table I; Fig. 1). Moreover, the expression level of PTPRO before PCI was significantly higher compared with that after PCI in both groups $(\mathrm{P}<0.05$; Table I; Fig. 1). A significant association was observed between prognosis and time $(\mathrm{F}=3217.922 ; \mathrm{P}<0.001)$, namely, the level of reduction about PTPRO in the good prognosis group was more obvious than that in the poor prognosis group ( $\mathrm{P}<0.05$; Table I and Fig. 1).

Prognostic efficacy of PTPRO expression in patients with $A C S$. The area under the ROC curve (AUC), maximum approximation index, optimal cut-off point, sensitivity, and specificity for the ability of the pre-PCI PTPRO expression level to predict the prognosis of patients with ACS were 0.536 [95\% confidence interval (CI), 0.462-0.610], 0.095, 1.08, 36.67 and $72.80 \%$, respectively. These indexes for the post-PCI PTPRO expression level were 0.883 (95\% CI, 0.828-0.926), $0.661,0.44,73.33$ and $92.80 \%$, respectively. The AUC value for PTPRO expression after PCI was significantly higher compared with hat for PTPRO expression before PCI ( $Z=6.714$; $P<0.001$; Fig. 2).
Comparison of characteristics between patients with high and low PTPRO expression after PCI. According to the optimal cutoff point for evaluating the prognosis of patients with ACS after PCI, patients with ACS were divided into a high PTPRO expression group $(\mathrm{n}=53)$ and a low PTPRO expression group $(n=132)$. No statistically significant differences were observed between the two groups in terms of age, sex, BMI, medical history (hypertension, diabetes, hyperlipidemia), family history of ACS, clinical type of ACS, and location and number of vascular lesions $(\mathrm{P}>0.05$; Table II).

Factors influencing prognosis of patients with ACS treated with PCI. In the high PTPRO expression group, 44 MACEs occurred, including 20 cases of RMI, 11 cases of RUA, 8 cases of stent restenosis, 3 cases of TVR, and 2 cases of cardiac death. In the low PTPRO expression group, 16 MACEs occurred, including 6 cases of RMI, 3 cases of RUA, 4 cases of stent restenosis, 2 cases of TVR, and 1 case of cardiac death. The median disease-free survival time of the high PTPRO expression group (20 months) was significantly shorter compared with that of the low PTPRO expression 
Table II. Comparison of patient characteristics between groups according to PTPRO expression after PCI.

\begin{tabular}{|c|c|c|c|c|}
\hline Item & High PTPRO group $(n=53)$ & Low PTPRO group $(n=132)$ & $t / \chi^{2}$ & P-value \\
\hline Age, years & $55.15 \pm 9.02$ & $55.58 \pm 8.71$ & 0.297 & 0.767 \\
\hline \multicolumn{5}{|l|}{ Sex } \\
\hline Male & 36 & 94 & \multirow[t]{2}{*}{0.196} & \multirow[t]{2}{*}{0.658} \\
\hline Female & 17 & 38 & & \\
\hline $\mathrm{BMI}, \mathrm{kg} / \mathrm{m}^{2}$ & $26.88 \pm 3.44$ & $26.56 \pm 2.87$ & 0.643 & 0.521 \\
\hline Hypertension & 26 & 61 & 0.123 & 0.726 \\
\hline Hyperlipidemia & 28 & 63 & 0.394 & 0.530 \\
\hline $\mathrm{T} 2 \mathrm{DM}$ & 18 & 40 & 0.235 & 0.628 \\
\hline ACS family history & 7 & 7 & 3.378 & 0.066 \\
\hline \multicolumn{5}{|l|}{ ACS type } \\
\hline STEMI & 14 & 33 & \multirow[t]{3}{*}{0.053} & \multirow[t]{3}{*}{0.974} \\
\hline NSTEMI & 26 & 67 & & \\
\hline UA & 13 & 32 & & \\
\hline \multicolumn{5}{|l|}{ Vascular lesion count } \\
\hline Single & 17 & 53 & \multirow[t]{2}{*}{1.049} & \multirow[t]{2}{*}{0.306} \\
\hline Multiple & 36 & 79 & & \\
\hline \multicolumn{5}{|c|}{ Vascular lesion location } \\
\hline LMCA & 6 & 9 & \multirow[t]{4}{*}{1.238} & \multirow[t]{4}{*}{0.744} \\
\hline LAD & 28 & 78 & & \\
\hline LCX & 8 & 19 & & \\
\hline RCA & 11 & 26 & & \\
\hline
\end{tabular}

PTPRO, protein tyrosine phosphatase receptor-type O; BMI, body mass index; T2DM, type 2 diabetes mellitus; ACS, acute coronary syndrome; STEMI, ST-elevation acute myocardial infarction; NSTEMI, non-ST-elevation acute myocardial infarction; UA, unstable angina; LMCA, left main coronary artery; LAD, left anterior descending; LCX, left circumflex; RCA, right coronary artery.

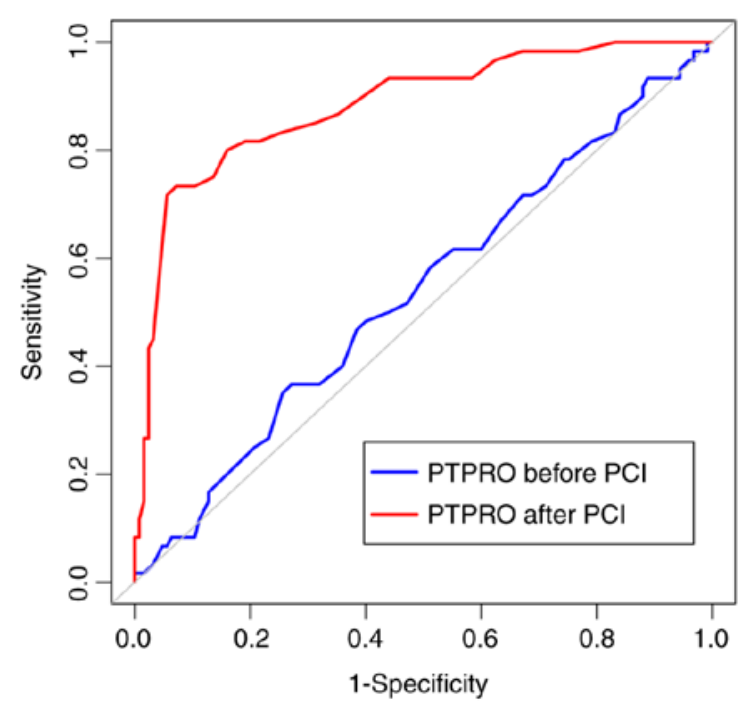

Figure 2. Receiver operating characteristic curve for the ability of PTPRO to predict the prognosis of patients with acute coronary syndrome. PTPRO, protein tyrosine phosphatase receptor-type $\mathrm{O}$.

group (36 months; log-rank $\chi^{2}=113.704 ; \mathrm{P}<0.001$; Fig. 3). For correlation analysis, age, sex, BMI, medical history (hypertension, diabetes, hyperlipidemia), family history of ACS, clinical type of ACS, location and number of vascular lesions, and the expression level of PTPRO were considered as independent variables, and the prognosis of patients with ACS was considered as the dependent variable. Cox univariate regression analysis showed that family history of ACS and the expression level of PTPRO were significantly associated with the prognosis of patients with ACS $(\mathrm{P}<0.05)$. Furthermore, Cox multivariate regression analysis identified high PTPRO expression as an independent risk factor for poor prognosis in patients with ACS $(\mathrm{P}<0.05$; Table III).

Association between PTPRO expression and prognosis of patients with ACS undergoing PCI. The Cox regression analysis with restrictive cubic spline model showed that the expression level of PTPRO was associated with the prognosis of patients with ACS $\left(\chi^{2}=51.48 ; \mathrm{P}<0.001\right)$ via a nonlinear association $\left(\chi^{2}=10.65 ; P=0.001\right)$. According to the cut-off point, the risk of poor prognosis decreased with a PTPRO expression level $<0.44$ but increased with a PTPRO expression level $>0.44$ (Fig. 4).

\section{Discussion}

The present study investigated the expression level of PTPRO in patients with ACS who underwent PCI and its association with prognosis. By comparing the expression level of PTPRO in peripheral blood mononuclear cells between groups with a 


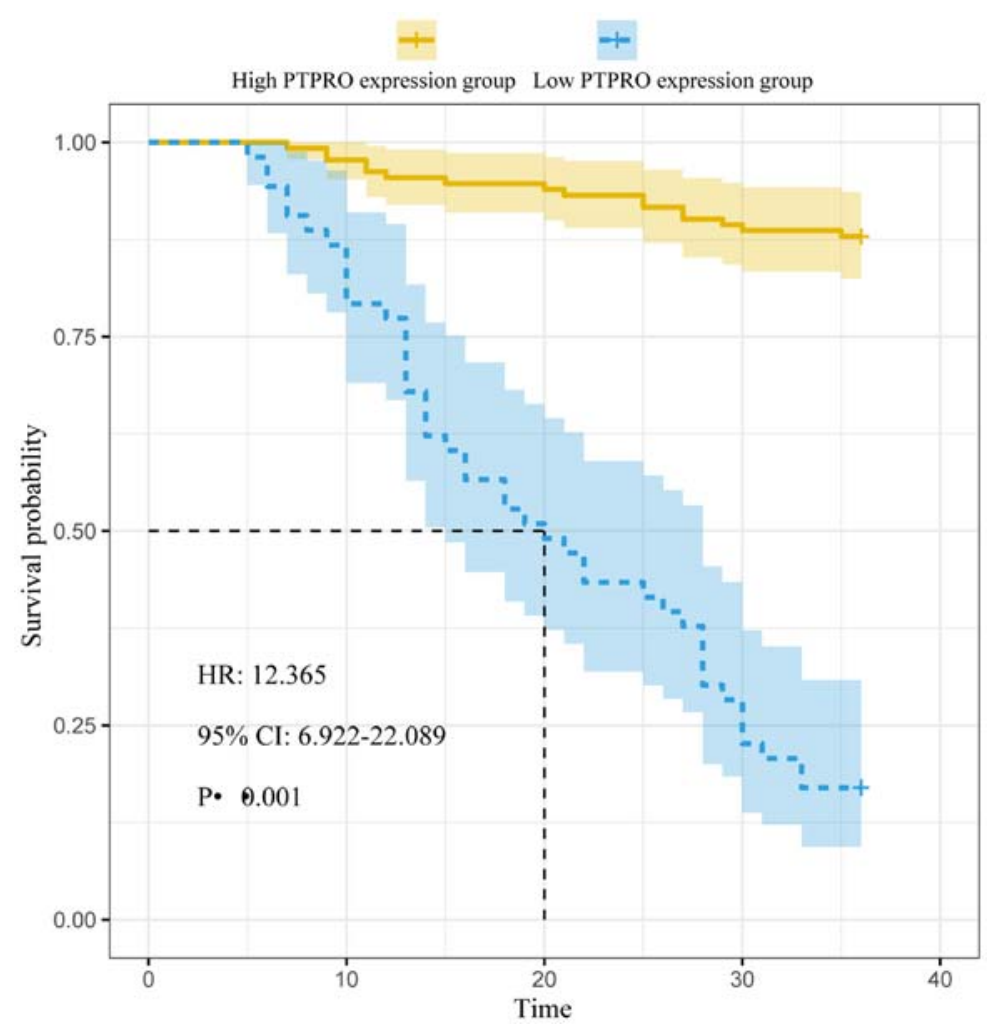

Figure 3. Kaplan-Meier disease-free survival curves for patients with acute coronary syndrome according to prognosis. PTPRO, protein tyrosine phosphatase receptor-type $\mathrm{O}$; HR, hazard ratio; $\mathrm{CI}$, confidence interval.

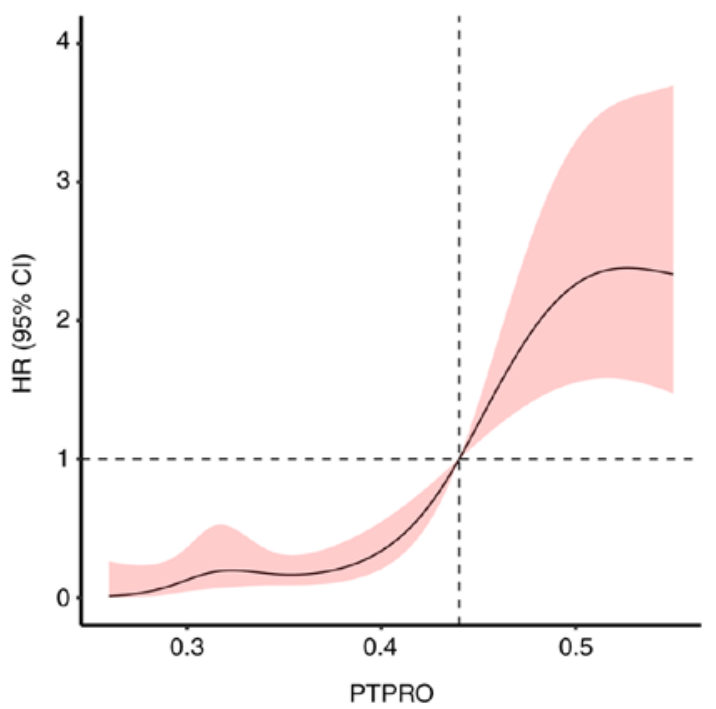

Figure 4. Results of Cox analysis with restricted cubic spline model for the correlation between the prognosis of patients with ACS and PTPRO expression. ACS, acute coronary syndrome; PTPRO, protein tyrosine phosphatase receptor-type $\mathrm{O}$.

good prognosis and a poor prognosis, it was found that the expression levels of PTPRO in both groups after PCI were lower compared with those before PCI, and the post-PCI expression level of PTPRO in the good prognosis group was lower compared with those in the poor prognosis group. These results indicate that PTPRO is involved in the pathogenesis and progression of ACS, and a lower expression level of PTPRO after PCI might be associated with improvement in the inflammatory response and decreased oxidative stress. Therefore, the expression level of PTPRO may contribute to the evaluation of prognosis in these patients.

Atherosclerosis is the pathological basis of ACS. Recently, several studies have suggested that atherosclerosis is not a condition of simple lipid deposition but a chronic inflammatory disease $(15,16)$. Inflammation plays a key role in the pathogenesis and progression of ACS (15-17), by mediating intracoronary thrombosis, atherosclerotic plaque damage, erosion and rupture (18). Mononuclear cells in peripheral blood also have a crucial role in the development of atherosclerosis through activating vascular endothelial cells, as well as releasing inflammatory mediators, such as interleukin-1 and C-reactive protein (19).

An elevated serum hypersensitive C-reactive protein level is an important predictor of cardiovascular events (20). After treatment with anti-interleukin-1 $\beta$ in patients with atherosclerosis, the serum C-reactive protein level was significantly decreased, and the incidence of cardiovascular events were subsequently decreased (21). However, the efficacy of C-reactive protein for predicting the prognosis of patients with ACS seemed to be limited, according to the finding that the AUC value for the prognostic value of C-reactive protein was only 0.634 (22). In the present study, the prognostic value of the PTPRO expression level in peripheral blood mononuclear cells in patients with ACS was investigated, and the results suggest that PTPRO expression in peripheral blood mononuclear cells after PCI can be used for the prediction of prognosis in patients with ACS.

To further clarify the association between the expression level of PTPRO and the prognosis of patients with ACS, the 
Table III. Cox regression analysis of factors affecting the prognosis of patients with ACS.

\begin{tabular}{|c|c|c|c|c|c|c|}
\hline \multirow[b]{2}{*}{ Item } & \multicolumn{3}{|c|}{ COX single-factor analysis } & \multicolumn{3}{|c|}{ COX multi-factor analysis } \\
\hline & HR & $95 \% \mathrm{CI}$ & P-value & HR & $95 \% \mathrm{CI}$ & P-value \\
\hline Age & 1.006 & $0.977-1.036$ & 0.691 & & & \\
\hline Sex & 1.108 & $0.634-1.937$ & 0.721 & & & \\
\hline BMI & 1.104 & $0.932-1.103$ & 0.743 & & & \\
\hline Hypertension & 1.252 & $0.757-2.073$ & 0.384 & & & \\
\hline Hyperlipidemia & 0.885 & $0.534-1.466$ & 0.636 & & & \\
\hline T2DM & 1.124 & $0.659-1.918$ & 0.669 & & & \\
\hline ACS family history & 2.261 & $1.077-4.746$ & 0.032 & 1.291 & $0.611 \sim 2.725$ & 0.505 \\
\hline ACS type & 1.069 & $0.745-1.534$ & 0.718 & & & \\
\hline Vascular lesion count & 1.075 & $0.638-1.813$ & 0.786 & & & \\
\hline Vascular lesion location & 1.069 & $0.811-1.409$ & 0.638 & & & \\
\hline High PTPRO expression & 12.365 & $6.922 \sim 22.089$ & $<0.001$ & 12.084 & $6.733 \sim 21.689$ & $<0.001$ \\
\hline
\end{tabular}

ACS, acute coronary syndrome; BMI, body mass index; T2DM, type 2 diabetes mellitus; PTPRO, protein tyrosine phosphatase receptor-type O.

median disease-free survival times was compared between patients with high and low PTPRO expression according to the calculated cutoff value. The results showed that the median disease-free survival time was shorter in those with high PTPRO expression compared with those with low PTPRO expression, which further indicates that the expression level of PTPRO is associated with the prognosis of patients with ACS. Cox regression analysis identified high PTPRO expression as an independent risk factor for poor prognosis in patients with ACS. In addition, Cox regression analysis with the restrictive cubic spline model showed an increased risk of poor prognosis with a PTPRO expression level $>0.44$, using a cutoff value of PTPRO expression $=0.44$. All of the results from the present study indicated that PTPRO expression after PCI is associated with the prognosis of patients with ACS.

Previous studies showed that PTPRO is involved in the inflammatory response and oxidative stress. For example, PTPRO was shown to activate NF- $\kappa \mathrm{B}$ in the regulation of hepatic ischemia-reperfusion injury through feedback mechanisms (23) and to promote the oxidative stress and cellular apoptosis by inducing oxidized low-density lipoprotein via the TLR4/NF- $\kappa B$ signaling pathway (11). It was speculated that the underlying reason might be that PTPRO participates in the atherosclerotic inflammation and oxidative stress response through the TLR4/NF- $\mathrm{KB}$ signal transduction pathway, which further promotes the damage, erosion and rupture of atherosclerosis plaques, and subsequently induces ACS. The expression level of PTPRO in patients with ACS could be detected following PCI, in order to understand the status of oxidative stress and inflammation, which further reflects the prognosis of patients with ACS.

In summary, the expression level of PTPRO in peripheral blood mononuclear cells after PCI was shown to be associated with the prognosis of patients with ACS. High PTPRO expression indicated a high risk of poor prognosis in patients with ACS undergoing PCI. Further prospective, multi-center, large-scale trials are needed to verify the results of the present study.

\section{Acknowledgements}

None.

\section{Funding}

No funding was received.

\section{Availability of data and materials}

The datasets generated and/or analyzed during the current study are available from the corresponding author on reasonable request.

\section{Authors' contributions}

MH designed the study, analyzed the data and wrote the manuscript; MG diagnosed the disease, supervised the study and revised the manuscript; RY collected the data, isolated mononuclear cells and detected protein tyrosine phosphatase. All authors read and approved the final manuscript. MH and MG confirm the authenticity of all the raw data.

\section{Ethics approval and consent to participate}

The study was approved by the medical ethics committee of Beijing Lube Hospital, Capital Medical University (approval no. 2016-LHKY-012-013). All procedures performed in studies involving human participants were in accordance with the ethics standards of the institutional and national research committee and with the 1964 Helsinki Declaration and its later amendments or comparable ethics standards. Written informed consent was obtained from all individual participants included in the present study. 


\section{Patient consent for publication}

Not applicable.

\section{Competing interests}

The authors declare that they have no competing interests.

\section{References}

1. Lin F, Yang Y, Guo Q, Xie M, Sun S, Wang X, Li D, Zhang G, Li M, Wang $J$ and Zhao G: Analysis of the molecular mechanism of acute coronary syndrome based on circRNA-miRNA network regulation. Evid Based Complement Alternat Med 2020: 1584052, 2020.

2. Chandiramani R, Cao D and Mehran R: Periprocedural anticoagulation in non-ST-segment elevation acute coronary syndrome: Time to reassess? Ann Transl Med 8: 556, 2020.

3. Huang ZS, Zheng ZD, Zhang JW, Tang LL, Zhou LL, Li SH Xie XJ, Dong RM, Zhu JM and Liu JL: Association of major adverse cardiovascular events and cardiac troponin-I levels following percutaneous coronary intervention: A three-year follow-up study at a single center. Eur Rev Med Pharmacol Sci 24: 3981-3992, 2020.

4. Ming F and Sun Q: Epigenetically silenced PTPRO functions as a prognostic marker and tumor suppressor in human lung squamous cell carcinoma. Mol Med Rep 16: 746-754, 2017.

5. Dong H, Ma L, Gan J, Lin W, Chen C, Yao Z, Du L, Zheng L, Ke C, Huang X, et al: PTPRO represses ERBB2-driven breast oncogenesis by dephosphorylation and endosomal internalization of ERBB2. Oncogene 36: 410-422, 2017.

6. Yan S, Cheng M, Duan Q, Wang Z, Gao W, Ren B and Xu D: MiR-6803-5p promotes cancer cell proliferation and invasion via $\mathrm{PTPRO} / \mathrm{NF}-\mathrm{\kappa B}$ axis in colorectal cancer. Mediators Inflamm 2019: 8128501, 2019.

7. Xu D, Wang $X$, Yan S, Yin Y, Hou J, Wang X and Sun B: Interaction of PTPRO and TLR4 signaling in hepatocellular carcinoma. Tumour Biol 35: 10267-10273, 2014.

8. Qi C, Shao Y, Liu X, Wang D and Li X: The cardioprotective effects of icariin on the isoprenaline-induced takotsubo-like rat model: Involvement of reactive oxygen species and the TLR4/NF- $\kappa$ B signaling pathway. Int Immunopharmacol 74: $105733,2019$.

9. Tang YL, Jiang JH, Wang S, Liu Z, Tang XQ, Peng J, Yang YZ and $\mathrm{Gu} \mathrm{HF}$ : TLR4/NF- $\mathrm{BB}$ signaling contributes to chronic unpredictable mild stress-induced atherosclerosis in ApoE-/mice. PLoS One 10: e0123685, 2015.

10. Zhao J, Yan S, Zhu X, Bai W, Li J and Liang C: PTPRO exaggerates inflammation in ulcerative colitis through TLR4/NF- $\mathrm{KB}$ pathway. J Cell Biochem 121: 1061-1071, 2020.

11. Liang C, Wang X, Hu J, Lian X, Zhu T, Zhang H, Gu N and Li J: PTPRO promotes oxidized low-density lipoprotein induced oxidative stress and cell apoptosis through toll-like receptor 4/nuclear factor $\kappa B$ pathway. Cell Physiol Biochem 42: 495-505, 2017.
12. Chinese Medical Association Emergency Physicians Branch, Chinese Medical Association Cardiovascular Medicine Branch and Chinese Medical Association Laboratory Medicine Branch: Guidelines for the rapid diagnosis and treatment of acute coronary syndrome in the emergency department. Chin J Emerg Med 25: 397-404, 2016.

13. Zhao JW, Shi G, Ping JD and Ming L: Study on the negative regulation of TIPE2 from peripheral blood mononuclear cells on tissue factor in patients with bronchial asthma. Zhonghua Yi Xue Za Zhi 98: 2889-2893, 2018 (In Chinese).

14. Ye G, Qin Y, Wang S, Pan D, Xu S, Wu C, Wang X, Wang J, Ye H and Shen H: Lamcl promotes the Warburg effect in hepatocellular carcinoma cells by regulating PKM2 expression through AKT pathway. Cancer Biol Ther 20: 711-719, 2019.

15. Gowdak LHW: Atherosclerosis, inflammation, and genetics- and you thought it was just LDL-cholesterol. Arq Bras Cardiol 114: 273-274, 2020.

16. Frodermann V, Rohde D, Courties G, Severe N, Schloss MJ, Amatullah H, McAlpine CS, Cremer S, Hoyer FF, Ji F, et al: Exercise reduces inflammatory cell production and cardiovascular inflammation via instruction of hematopoietic progenitor cells. Nat Med 25: 1761-1771, 2019.

17. Zhang $\mathrm{Y}$, Zhang $\mathrm{C}$ and Zhang $\mathrm{M}$ : A new era of anti-inflammatory therapy for atherosclerosis. Zhonghua Xin Xue Guan Bing Za Zhi 46: 332-337, 2018 (In Chinese).

18. Sun YF and Guo DL: Research progress of the relationship between inflammatory factor and coronary heart disease. China Mod Med 24: 12-15, 2017.

19. Szekely Y and Arbel Y: A review of interleukin-1 in heart disease: Where do we stand today? Cardiol Ther 7: 25-44, 2018.

20. Gao S, Shu S, Wang L, Zhou J and Yuan Z: Pro-inflammatory and anti-inflammatory cytokine responses of peripheral blood mononuclear cells in apparently healthy subjects. Nan Fang Yi Ke Da Xue Xue Bao 34: 1589-1593, 2014 (In Chinese).

21. Ridker PM, Everett BM, Thuren T, MacFadyen JG, Chang WH, Ballantyne C, Fonseca F, Nicolau J, Koenig W, Anker SD, et al: Antiinflammatory therapy with canakinumab for atherosclerotic disease. N Engl J Med 377: 1119-1131, 2017.

22. Dongxu H, Yundi J, Jia S, Zongyu W, Jiake W, Weili D, Na S, Tongtong Y, Zhijun S and Zaoqing S: The effects of C-reactive protein and albumin ratio on the prognoses in patients with acute coronary syndrome. Chin J Cardiovasc Res 16: 1090-1094, 2018.

23. Hou J, Xia Y, Jiang R, Chen D, Xu J, Deng L, Huang X, Wang X and Sun B: PTPRO plays a dual role in hepatic ischemia reperfusion injury through feedback activation of NF- $\mathrm{BB}$. J Hepatol 60 : 306-312, 2014.

This work is licensed under a Creative Commons Attribution-NonCommercial-NoDerivatives 4.0 International (CC BY-NC-ND 4.0) License. 